\title{
Advantages of Scorpion Suture Passer and 70 Degrees Arthroscope in Arthroscopic Bankart Repair: Usefulness for Inferior Labral Repair
}

\author{
Yoon-Suk Hyun ${ }^{\varpi}$, Woo-Jin Shin \\ Department of Orthopaedic Surgery, Hallym University Kangdong Sacred Heart Hospital, Seoul, Korea
}

Background: The blunted tip of a reusable with multiple uses can cause problems with the passing procedure in arthroscopic Bankart repair. This study assessed the advantage of Scorpion with a $70^{\circ}$ arthroscope in arthroscopic Bankart repair compared to hook typed suture passer.

Methods: Scorpion in 19 patients, the hook type suture passer (conventional group) in 18 patients were used. All patients underwent the same procedure except for the type of suture passer used. Another different point of the procedure were telescopes and the number of portals used; three arthroscopic portals (posterior, anterorsuperiorlateral, and mid-anterior) and a $30^{\circ}$ arthroscope in the conventional group, but two portals and a $70^{\circ}$ arthroscope as well as the $30^{\circ}$ one in the Scorpion group. The surgery time and the surgical complications including an iatrogenic axillary nerve injury were recorded.

Results: The Scorpion group showed a significant decrease in surgery time compared to the conventional group. In contrast to the conventional group, Scorpion provided an easy estimation of the exit of suture passing, no iatrogenic labral injury during the passing procedure with straight movement and the sharp tip of the knife installed. latrogenic supraspinatus injuries could be avoided when making an accessory anteosuperiorlateral portal due to the $70^{\circ}$ arthroscope.

Conclusions: In arthroscopic Bankart repair, the use of the Scorpion suture passer and a $70^{\circ}$ arthroscope can reduce the surgery time, avoid unnecessary supraspinatus injury, and avoid iatrogenic axillary nerve damage through the relatively easy and precise suture passing and saving of the anterosuperior portal.

(Clin Shoulder Elbow 2017;20(4):201-207)

Key Words: Bankart lesion; Arthroscopy

\section{Introduction}

Arthroscopic Bankart repair has attracted increasing since its introduction almost 30 years ago because of the improved arthroscopic equipment and increased experience of surgeons. ${ }^{1-3)}$ Several techniques for the arthroscopic repair of antero-inferior labral tears have been introduced. ${ }^{4-11)}$ Among them, techniques using three portals (posterior, anterior-superior, and mid-anterior) are popular and easy to follow for trainee surgeons. ${ }^{4,10)}$ An anterosuperolateral portal is commonly used during anchor placement for superior labrum anterior to posterior (SLAP) repair or as a secondary viewing portal during Bankart repair. ${ }^{12)}$ This portal was originally placed in the rotator interval, but it can be made in the anterior part of the supraspinatus, as in the transrotator cuff approach, particularly in Asian patients or relatively small patients. ${ }^{13)}$ Therefore, an iatrogenic injury on the anterior part of the supraspinatus can be possible, even though previous studies of the transrotator cuff approach supported its safety. ${ }^{14-16)}$

Because iatrogenic axillary nerve injury can be possible during the arthroscopic intraarticular procedure, especially in inferior capsulolabral area, several studies have provided safe distances for preventing this complication. ${ }^{17-20)}$

In arthroscopic Bankart repair, several types of suture shuttles have been used depending on the surgeon's preferences. The

Received September 30, 2017. Revised November 11, 2017. Accepted November 13, 2017.

Correspondence to: Yoon-Suk Hyun

Department of Orthopaedic Surgery, Hallym University Kangdong Sacred Heart Hospital, 150 Seongan-ro, Gangdong-gu, Seoul 05355, Korea Tel: +82-2-2224-2230 1, Fax: +82-2-489-4391, E-mail: y1205s@hallym.or.kr

IRB approval (No. KANGDONG 2017-11-009).

Financial support: None. Conflict of interests: None. 
reusable hook typed suture passing system is the most popular type, but multiple reuse blunts the sharp edge of the tip of the suture passer and surgeons can encounter some problems related to passing the blunted suture passer through the detached hard inferior capsulolabral complex (Fig. 1).

In arthroscopic rotator cuff repair, a scorpion suture passer, which is a popular suture punch type shuttle device, has been used instead of the conventional hook type suture passer for the same problem related to blunted tips with reuse despite the paucity of reports. In addition, there are no reports of the use of Scorpion type suture shuttles in arthroscopic Bankart repair. $70^{\circ}$ arthroscope can save the accessory anterosuperiorlateral portal becasue of its wide vision angle compared to $30^{\circ}$ arthroscope. This study assessed the advantages of the Scorpion suture passer with $70^{\circ}$ arthroscope in arthroscopic Bankart or antero-inferior labral repair.

\section{Methods}

A prospective study of consecutive arthroscopic Bankart procedures, in which the Scorpion suture passer had been used began in 2014. Informed consent was obtained from all patients, and the institutional review board at all sites approved the research protocol. This study was a consecutive case control series of patients treated at the Hallym University Kangdong Sacred Heart Hospital from March 2014 to August 2016. Thirty seven patients were included. The preoperative data collected included the demographic information, preoperative symptoms, and physical examination. The postoperative data collected included functional questions, pain levels, number of dislocations, physical examinations, and radiographs. A pre- and postoperative Constant score and Rowe score were determined. ${ }^{21,22)}$ In the present study, the maximum Constant score was 75 because the abduction power could not evaluated. The data (at 6 - to

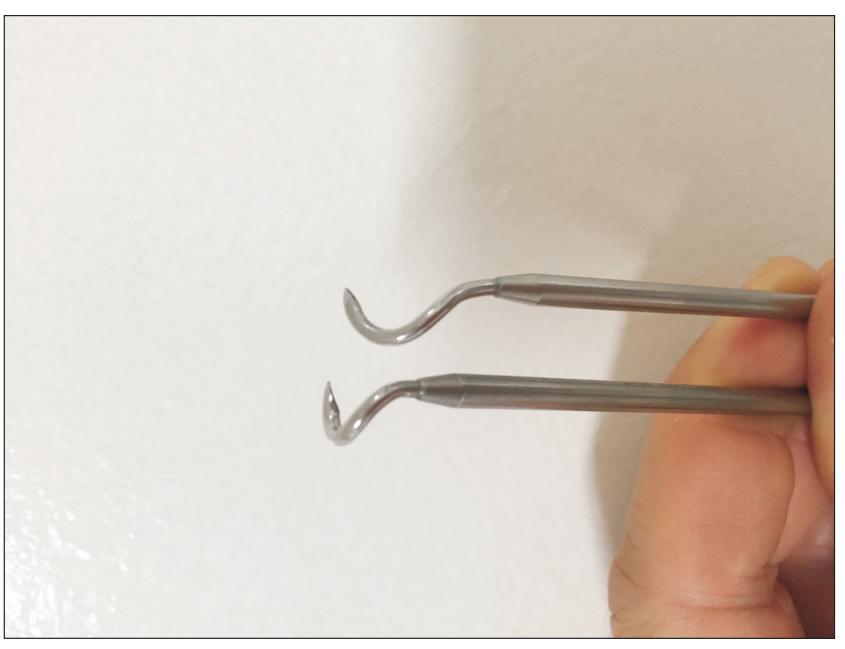

Fig. 1. Sharp tip of the hook became blunted with multiple reuse. 12-month intervals) were collected at each visit.

The inclusion criteria were one or more traumatic dislocations or multiple posttraumatic subluxations with anteroinferior labral tear suspected with magnetic resonance imaging, adequate bone, and no prior shoulder surgery. The exclusion criteria were recent infection; bilateral, voluntary, or atraumatic instability; adhesive capsulitis; nerve injury; posterior or multidirectional instability; significant glenoid deficiency; large Hill-Sachs lesions; bony Bankart lesion; or rotator cuff tears.

The Scorpion suture passer was used in 19 patients (Scorpion group), and the conventional hook type suture passer was used in 18 patients (conventional group). All 37 patients underwent the same arthroscopic procedure except for the type of suture passer used. Other differences in the procedure were telescopes and the number of portals used; three arthroscopic portals (posterior, anterorsuperiorlateral, and mid-anterior) and $30^{\circ}$ angled telescope were necessary in patients in the conventional group, whereas two portals (posterior and mid anterior) and $70^{\circ}$ angled telescope as well as the $30^{\circ}$ one were used in the Scorpion group. To avoid confounding factors with the suture anchor and knot tying method, three suture anchors with the same type and the same type of knot tying (Revo) were used in all 37 patients. The surgery time and complications related to the surgical technique including the incidence of iatrogenic axillary nerve injury were recorded.

\section{Surgical Procedure in Scorpion Group}

The patient was placed in the lateral decubitus position with the index arm in a pneumatic arm positioner (SPIDER2, Smith \& Nephew or AssistArm, ConMed). After an examination under anesthesia to assess the grade of instability, a standard posterior portal for arthroscopy was established. The diagnostic arthroscopy with the $30^{\circ}$ arthroscope evaluated all aspects of the glenohumeral joint and the presence of a Bankart lesion was confirmed and other pathologies were noted. An anterior portal was established with a 5.0-mm cannula (Arthrex, Naples, FL, USA) in the rotator interval, and the Bankart lesion was assessed with a probe. A 5.0-mm cannula in anterior portal was changed to an $8.25-\mathrm{mm}$ cannula (Arthrex) before bony preparation. A $70^{\circ}$ arthroscope was switched to visualize and evaluate the status of the inferior labrum. The instruments were placed through the anterior portals to mobilize the Bankart lesion and the entire inferior glenohumeral ligament (IGHL), as a sleeve of continuous tissue, was placed inferiorly to the 6-o'clock position. The glenoid neck was prepared using an elevator and a bone rasp to for the bleeding surfaces to enhance bone-to- bone healing. The curved drilling guide was placed into the joint at approximately the 5 o'clock position ( 7 o'clock for left arm). The first or inferior hole was drilled at the edge of the glenoid articular surface by 1.3 $\mathrm{mm}$ drill and $1.8 \mathrm{~mm}$ Y-Knot Flex All-Suture Anchors (ConMed, Largo, FL, USA), which are single-loaded with a \#2 Hi-Fi Suture 
were inserted.

The cannula of the anterior portal was then removed and reinserted. Both suture limbs were moved from inside the cannula to the outside. Only one suture limb was pulled out through the cannula of the anterior portal using a suture retriever and another limb was then located outside of the cannula. The suture limb in the cannula of the anterior portal was loaded into the slot on the bottom jaw of the Scorpion suture passer and the Scorpion was then advanced through the anterior cannula and moved to the detached capsulo-labral tissue at the 6 o'clock position for upward shifting of the inferior capsule-labral complex and IGHL. For easy approach to the inferior capsulo-labral tissue with a large Scorpion, the assistant pushed the involved arm with arm holder backward because the humeral head could interfere with the approach to the inferior capsulo-labral area with a large Scorpion. The next step is most important. Because the distance from the slot on the bottom jaw to the crossing point with upper jaw could be calculated in advance (approximately $15 \mathrm{~mm}$ in Scorpion), suture passing more than $10 \mathrm{~mm}$ from edge of the detached labrum could be avoided (Fig. 2). The instrument was squeezed gently with the palm of the hand, advancing the needle and suture limb through the tissue at the 6 o'clock position. Scorpion passed and retrieved the suture limb all in a single step. The anterior cannula was removed and reinserted again, the suture limb located outside of the cannula was passed and another suture limb was retrieved in the cannula and passed through near the edge of the labrum for horizontal mattress configuration. The sutures were then tied with standard Revo knot tying. Each step was then repeated, a second and third anchor were then inserted in a similar manner at 3:30 and 2 o'clock position (8:30 and 10 o'clock of left arm).

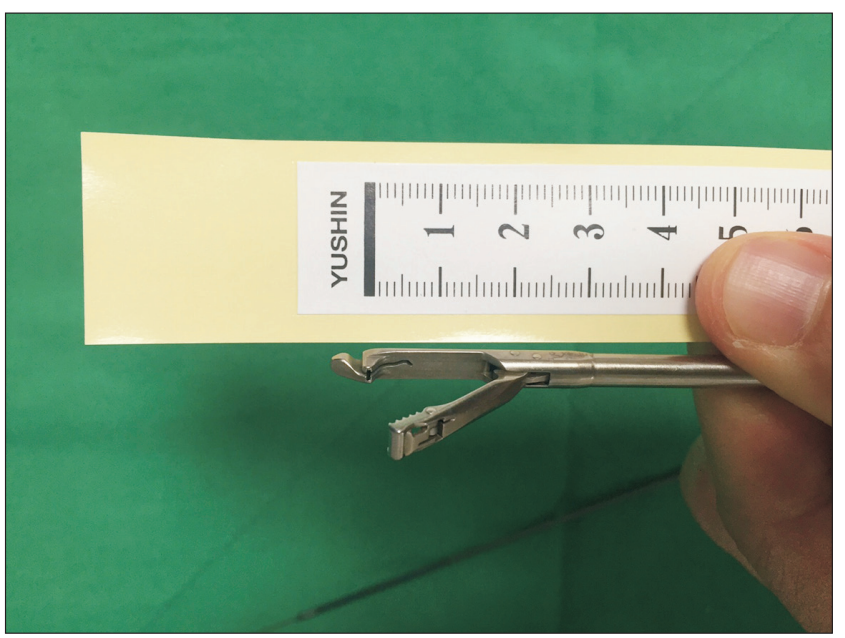

Fig. 2. Distance from the slot for the string load in the bottom jaw of the Scorpion suture passer to crossing point of two jaws is about $15 \mathrm{~mm}$.

\section{Different Points in the Conventional Group}

In the conventional group, an accessory anterosuperiorlateral portal was established through the anterior part of the supraspinatus muscle because two portals were necessary during the repair procedure with a conventional hook type suture passer. With visualization from the anterosuperior portal with a $30^{\circ}$ arthroscope, debridement of the labral edge of Bankart lesion and bony preparation procedure were done. Regarding suture passing, both limbs of the suture are passed through the soft tissues using the popular shuttling technique with a $45^{\circ}$ curved Suture hook (Spectrum; ConMed) with \#1 polydioxanone (PDS).

Suture configuration with horizontal mattress, count and positions of three anchors, and knot tying technique were same as in the Scorpion group.

The postoperative rehabilitation and follow-up protocol were the same in both groups. The Mann-Whitney U-test was used to evaluate the significance of the demographic data, surgery time, and preoperative Constant and Rowe scores between the 2 groups. The Wilcoxon signed rank test was used to assess the difference between the preoperative and postoperative shoulder scores. Data analysis was conducted using the SPSS software ver. 15.0 (SPSS Inc., Chicago, IL, USA). The level of statistical significance was set to $p<0.05$.

\section{Results}

Table 1 lists the demographic data. The number of dislocations, period from the last dislocation and surgery and age were similar in both groups. The Scorpion group showed a significant reduction of surgery time compared to the conventional group (Table 2). Regarding the clinical outcomes with Constant and Rowe scores, all 37 patients showed improvement and there was no significant difference in the preoperative, postoperative and improvement between the two groups (Table 3).

The Scorpion suture passer made it easy to pass the suture

Table 1. Demographic Factors

\begin{tabular}{lccc}
\hline \multicolumn{1}{c}{ Variable } & $\begin{array}{c}\text { Scorpion } \\
\text { group }\end{array}$ & $\begin{array}{c}\text { Conventional } \\
\text { group }\end{array}$ & -value \\
\hline No. of patient & 19 & 18 & - \\
\hline Sex (male:female) & $17: 2$ & $17: 1$ & - \\
\hline Age (yr) & $24.6(19-37)$ & $23.2(18-29)$ & 0.471 \\
\hline Dominant side involved & 16 & 16 & - \\
\hline No. of dislocations & $2.5(1-5)$ & $2.7(1-6)$ & 0.646 \\
\hline $\begin{array}{l}\text { Period from last dislocation } \\
\text { to surgery (mo) }\end{array}$ & $18.5(0-48)$ & $21.0(0-39)$ & 0.513 \\
\hline Follow-up periods (mo) & $15.6 \pm 2.7$ & $16.9 \pm 3.2$ & - \\
\hline
\end{tabular}

Values are presented as number only, median (range), or mean \pm standard deviation. 
Table 2. Surgery Time in Both Groups

\begin{tabular}{cc}
\hline Group & Surgery time \\
\hline Conventional group & $42.5 \pm 13.5$ \\
Scorpion group & $27.4 \pm 16.2$ \\
$p$-value & $<0.001$ \\
\hline
\end{tabular}

Values are presented as mean \pm standard deviation.

Table 3. Shoulder Scores

\begin{tabular}{|cccc}
\hline \multicolumn{1}{c}{ Score } & Preoperative & Last visit & Improvement \\
\hline Constant score & & & \\
\hline Scorpion & $46.4(35-55)$ & $65.6(55-75)$ & $19.2(4-40)$ \\
Conventional & $45.6(40-55)$ & $63.2(55-75)$ & $16.7(4-34)$ \\
\hline$p$-value & 0.558 & 0.210 & 0.408 \\
\hline Rowe score & & & $45(15-60)$ \\
\hline Scorpion & $43.9(35-55)$ & $88.7(55-100)$ & $43.3(15-60)$ \\
\hline Conventional & $43.9(35-55)$ & $86.7(55-100)$ & 0.845 \\
\hline$p$-value & 0.869 & 0.685 & \\
\hline
\end{tabular}

Values are presented as median (range).

limb on the required positions quickly and there was no iatrogenic labral injury caused by the passing procedure of the instrument itself, which is in contrast to that observed in the conventional group, in which iatrogenic labral injury occurred frequently in many cases, even though injuries on suture passing were not too severe to spoil the surgeries (Fig. 3). The Scorpion suture passer did not require the use of a PDS or wire loop for real suture string passing. In the conventional group, however, PDS or wire loop snapped frequently whilst being passed though the hard labral tissue, even though the incidence was not recorded, and the bridge point between the curved part and straight part of the instrument broke in 2 out of 18 cases because the blunted tip after multiple uses made it more difficult to pass it through the labrum without a stronger push force. None of the 37 patients showed axillary nerve injury after surgery or revisited with a recurrent dislocation during the follow-up period.

\section{Discussion}

The Scorpion suture passer and $70^{\circ}$ arthroscope allowed the arthroscopic Bankart repairs to be completed without an anterosuperiorlateral portal and with a decrease in surgery time. latrogenic axillary nerve injury was not a concern while passing the suture through the infeior capsulo-labral complex.

The Scorpion suture passer (Arthrex) is not new equipment. Several similar suture punch types are also available on the market: Truepass and Firstpass (Smith \& Nephew, London, UK), Expressew (Mitek; DePuy Synthes, Raynham, MA, USA),

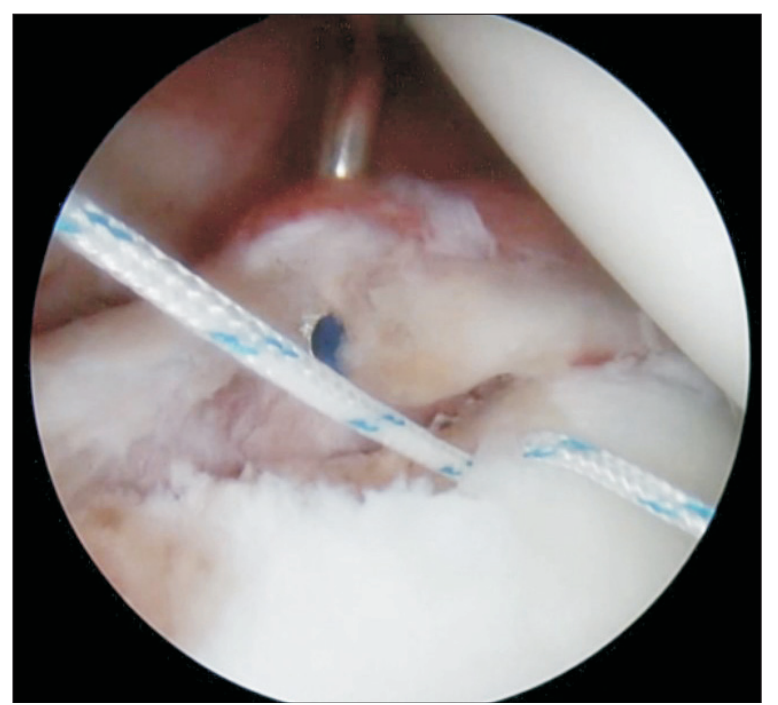

Fig. 3. Blunted tip of the curved hook type suture passer can cause iatrogenic labral injury during the passing procedure of the instrument.

and Spectrum Autopass (ConMed). These devices are modified forms of the Caspari suture punch (Linvatec; ConMed). The Caspari suture punch has been used in several studies on Caspari transglenoid Bankart repair. ${ }^{5-7,23,24)}$ These suture punch type shuttle devices, which use a disposable sharp knife have been used well in arthroscopic rotator cuff repair for several years in the authors' clinic and it is believed that this device can be used also in arthroscopic Bankart repair instead of reusable shuttle devices because the blunted tip with multiple use made it difficult to perform the suture passing procedure.

Among the reusable suture shuttles, Suture Lasso (Arthrex) and Spectrum series (ConMed) can be thought as the most versatile arthroscopic suture passing system on the market. On the other hand, these spiral shaped reusable shuttle systems also have reusable suture hooks. Continuous reuse blunts the sharp edge of the tip of these hooks and surgeons can experience difficulty in passing a blunted suture passer through the labrum or rotator cuff. Capsulo-labral tissue can be torn with a passing procedure and the bridging part between the shaft and hook can be broken because of the excessive power, which make surgery more complicated.

Nerve injury is a commonly reported complication in arthroscopic labral repair with the axillary nerve being the most frequently involved $(0.3 \%) .{ }^{25,26)}$ According to recent studies, the average distance between the axillary nerve and inferior glenoid rim was 10 to $25 \mathrm{~mm}$ in $45^{\circ}$ abduction with neutral rotation. ${ }^{18)}$ The axillary nerve can be injured when placing sutures at the anteroinferior and inferior positions. ${ }^{20,25)}$ Care must be taken to avoid over-penetration with the suture passer around the inferior glenohumeral ligament, which could injure the adjacent axillary nerve. $^{27)}$ 
While passing through the inferior capsule-labral tissue with a spiral hook suture passer, the tip of the hook can pass a more inferior part than the entering point of the capsule through its spiral movement. Moreover, an estimation of the appropriate entering and exit point in the surgical field to avoid iatrogenic axillary injury cannot be easy with a spiral suture hook, particularly in inexperienced surgeons because the hook part of each type has a variable length (Fig. 4).

The blunted tip of the hook with reuse, possible passing through the deep part near the axillary nerve with spiral movement and difficulty in estimating the appropriate entering point in inexperienced surgeons can increase the likelihood of iatrogenic injury to the axillary nerve around the inferior capsule, despite this being very rare. ${ }^{25)}$

The needle for suture passing of Scorpion is disposable and sharper than the tip of a hook, and there is no concern about a blunted tip. The movement of the needle in the Scorpion suture passer is straight. The distance from the needle slot on the bottom jaw of Scorpion to the dividing point of jaws is approximately $15 \mathrm{~mm}$. The likelihood of iatrogenic axillary nerve injury can be reduced using the Scorpion type suture passer because an estimation of the appropriate entering and exit point can be easier than a hook type suture passer.

In the three portal technique with a spiral hook type suture passer, the PDS or wire loop installed in suture passer should be passed through the torn capsule-labral tissue before real suture passing. For a connection between the real suture string attached to the suture anchor and PDS or wire loop of the suture passer, an additional working portal is necessary except for the viewing portal. In this procedure, one working portal is sufficient because suture passing with the scorpion type of suture passer does not require that connection, which can reduce the surgery

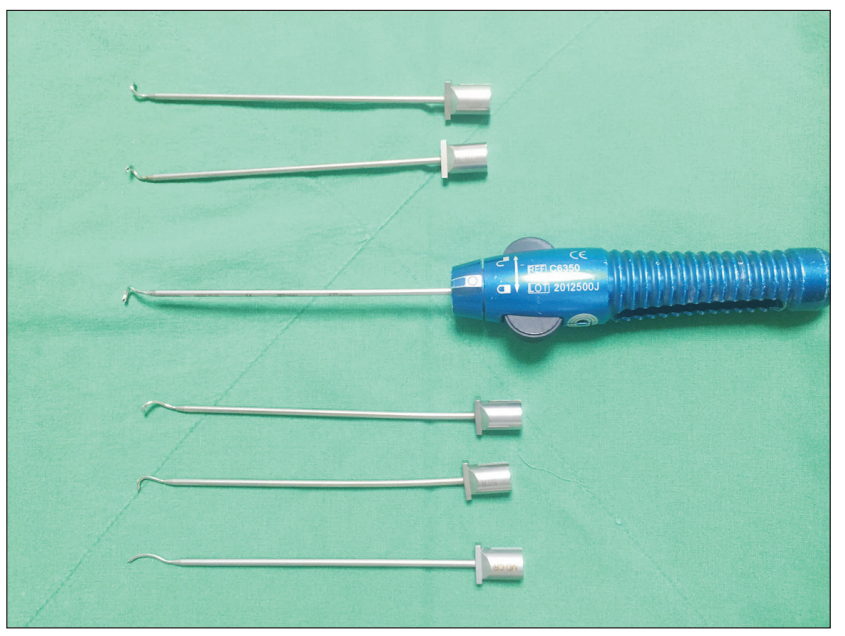

Fig. 4. Length of the hook part of each type are variable and screw movement can make it difficult to estimate the exit of the instrument during the passing procedure. time. Easy suture passing could also be helpful in reducing the surgery time.

In particular, around the 6 o'clock position, the exit point of the hook tip could not be estimated easily and screw movement was sometimes impeded by the humeral head in patients with tight joint space in the conventional group because suture passing with a curved hook suture passer can cause screw movement over the capsule. In suture passing with Scorpion, an estimation of the exit of the suture limb could be relatively easy because suture passing could be performed while observing the known-length bottom jaw of Scorpion and screw movement was not made (Fig. 5).

In addition, delicate passage and positioning of the suture strings can be possible, and an unnecessary injury to the capsulo-labral tissue through the passing procedure of the instrument itself can be avoided because the Scorpion needle is finer than a suture hook.

Regarding the use of the anterosuperiorlateral portal, Savoie et al. ${ }^{7)}$ used only two portals (standard anterior working and posterior viewing portals) and a Caspari suture punch and $30^{\circ}$ telescope in 1997. They also used an additional antero-superior portal for better access in an inferior labral detachment study in 1999. In recent studies for arthroscopic surgery for anterior instability, the use of the anterosuperiorlateral portal as well as anterior and posterior portals for the repair procedure is known as a routine. ${ }^{8,28)}$ An anterosuperolateral portal is commonly used during anchor placement for SLAP repair or as a secondary viewing portal during Bankart repair. ${ }^{2)}$ This portal was placed approximately $1 \mathrm{~cm}$ lateral to the anterolateral corner of the acromion and was established through the rotator interval just anterior to the supraspinatus tendon and posterior to the long

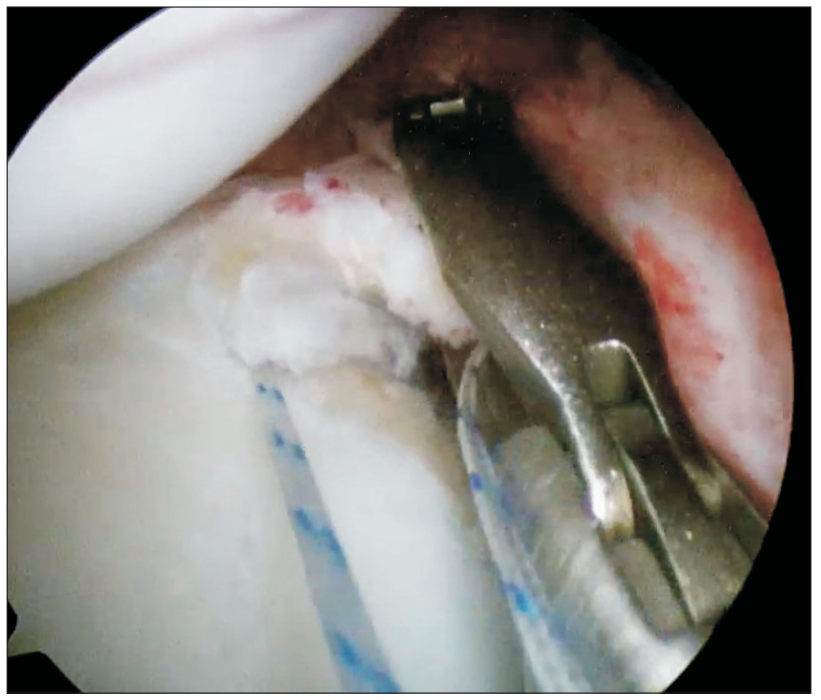

Fig. 5. Estimation of the exit of the suture limb could be relatively easy because suture passing could be performed while observing the known-length bottom jaw of the Scorpion suture passer. 
head of the biceps. ${ }^{12)}$ The anterosuperiorlateral portal is very useful as a viewing portal for evaluating the inferior labrum and release of capsulo-labral complex and decortication, and making a decision regarding an appropriate spot for suture anchor insertion for an inferior labral tear near the 6 o'clock point, particularly in patients with insufficient intra-articular space. This portal was originally placed in the rotator interval just anterior to the supraspinatus tendon and posterior to the long head of the biceps, but it can be made in the anterior part of the supraspinatus as in the transrotator cuff approach, especially in Asian patients or relatively small patients. ${ }^{13)}$ Therefore, iatrogenic injury to anterior part of supraspinatus is possible during the penetration of the cannula despite previous studies on the transrotator cuff approach supporting its safety. ${ }^{14-16)}$ In the three portals technique (anterior, antreo-superior and posterior portals) in arthroscopic Bankart repair with conventional suture shuttles with a spiral curved hook, the role of the antero-superior portal is the second viewing portal with a $30^{\circ}$ arthroscope and two additional portals are necessary during the repair procedure for an inferior labraum around the 6 o'clock area. An evaluation of the inferior labrum and preparation of the inferior glenoid through a standard posterior viewing portal with $30^{\circ}$ arthroscope is sometimes difficult in patients without severe laxity of the glenohumeral joint.

The $70^{\circ}$ arthroscope through standard posterior portal can allow an evaluation of the inferior labrum, regardless of the existence of severe glenohumeral laxity and only one anterior working portal is sufficient in the repair procedure with a Scorpion suture passer. The anterosuperiorlateral portal can be saved and the possibility of rotator cuff injury while making this portal can be reduced using a Scorpion suture passer and a $70^{\circ}$ arthroscope.

The advantages of using the Scorpion suture passer and $70^{\circ}$ arthroscope in arthroscopic Bankart repair can be summarized as follows:

1. latrogenic axillary nerve injury during suture passing on inferior labral repair can be prevented using the sharp needle tip of the Scorpion suture passer, straight movement of the Scorpion needle, and easy estimation of the penetration point of suture passing.

2. The possibility of iatrigenic suptaspinatus injury can be avoided by making an accessory anteosuperiorlateral portal.

3. The surgery time can be reduced through skipping unnecessary suture-shuttling procedures.

Scorpion also has a fatal limit to its use; it can break in the waist portion, which is a transitional zone with a knife shaft and sharp tip with multiple reuse. Because breakage of the knife tip usually occurs before the tip becomes blunted and follows the bending of the transitional zone of the knife, breakage of knife can be avoided if the knife of Scorpion can be changed into a new one when the bending on the waist zone of the disposable knife is noticed.

Although this study had several limitations with a non-randomized study and small sample size, the results are meaningful because this study identified the problem with the blunted tip of a hook type reusable suture passer, which used to be overlooked, and tried to use the Scorpion type suture passer for overcoming these problems.

\section{Conclusion}

In the arthroscopic Bankart repair, the use of a Scorpion suture passer and $70^{\circ}$ arthroscope can reduce the surgery time, avoid the unnecessary supraspinatus injury and provide a sense of relief to avoid iatrogenic axillary nerve through the relatively simple and precise suture passing and saving of the anterosuperior portal.

\section{References}

1. Green MR, Christensen KP. Arthroscopic versus open Bankart procedures: a comparison of early morbidity and complications. Arthroscopy. 1993;9(4):371-4.

2. Speer KP, Deng X, Borrero S, Torzilli PA, Altchek DA, Warren RF. Biomechanical evaluation of a simulated Bankart lesion. J Bone Joint Surg Am. 1994;76(12):1819-26.

3. Cole BJ, L'Insalata J, Irrgang J, Warner JJ. Comparison of arthroscopic and open anterior shoulder stabilization. A two to six-year follow-up study. J Bone Joint Surg Am. 2000;82(8): 1108-14.

4. Barber FA, Snyder SJ, Abrams JS, Fanelli GC, Savoie FH 3rd. Arthroscopic Bankart reconstruction with a bioabsorbable anchor. J Shoulder Elbow Surg. 2003;12(6):535-8.

5. Marcacci M, Zaffagnini S, Petitto A, Neri MP, lacono F, Visani A. Arthroscopic management of recurrent anterior dislocation of the shoulder: analysis of technical modifications on the Caspari procedure. Arthroscopy. 1996;12(2):144-9.

6. Yoneda M, Hayashida K, Izawa K, Shimada K, Shino K. A simple and secure anchoring system for Caspari's transglenoid multiple suture technique using a biodegradable poly-I-lactic acid button. Arthroscopy. 1996;12(3):293-9.

7. Savoie FH 3rd, Miller CD, Field LD. Arthroscopic reconstruction of traumatic anterior instability of the shoulder: the Caspari technique. Arthroscopy. 1997;13(2):201-9.

8. Sugaya H, Kon Y, Tsuchiya A. Arthroscopic Bankart repair in the beachchair position: a cannulaless method using an intraarticular suture relay technique. Arthroscopy. 2004;20 Suppl 2: 116-20.

9. Marquardt B, Witt KA, Liem D, Steinbeck J, Pötzl W. Arthroscopic Bankart repair in traumatic anterior shoulder instability using a suture anchor technique. Arthroscopy. 2006; 22(9):931-6. 
10. Stein T, Buckup J, Efe T, et al. Structural and clinical integrity of the rotator cuff in athletes after arthroscopic Bankart repair using the three-portal technique. Arch Orthop Trauma Surg. 2015;135(3):369-82.

11. Thal R, Nofziger M, Bridges M, Kim JJ. Arthroscopic Bankart repair using Knotless or BioKnotless suture anchors: 2- to 7-year results. Arthroscopy. 2007;23(4):367-75.

12. Lo IK, Lind CC, Burkhart SS. Glenohumeral arthroscopy portals established using an outside-in technique: neurovascular anatomy at risk. Arthroscopy. 2004;20(6):596-602.

13. Meyer M, Graveleau N, Hardy P, Landreau P. Anatomic risks of shoulder arthroscopy portals: anatomic cadaveric study of 12 portals. Arthroscopy. 2007;23(5):529-36.

14. Oh JH, Kim SH, Lee HK, Jo KH, Bae KJ. Trans-rotator cuff portal is safe for arthroscopic superior labral anterior and posterior lesion repair: clinical and radiological analysis of 58 SLAP lesions. Am J Sports Med. 2008;36(10):1913-21.

15. O'Brien SJ, Allen AA, Coleman SH, Drakos MC. The transrotator cuff approach to SLAP lesions: technical aspects for repair and a clinical follow-up of 31 patients at a minimum of 2 years. Arthroscopy. 2002;18(4):372-7.

16. Stephenson DR, Hurt JH, Mair SD. Rotator cuff injury as a complication of portal placement for superior labrum anteriorposterior repair. J Shoulder Elbow Surg. 2012;21(10):1316-21.

17. Loomer R, Graham B. Anatomy of the axillary nerve and its relation to inferior capsular shift. Clin Orthop Relat Res. 1989;(243):100-5.

18. Yoo JC, Kim JH, Ahn JH, Lee SH. Arthroscopic perspective of the axillary nerve in relation to the glenoid and arm position: a cadaveric study. Arthroscopy. 2007;23(12):1271-7.

19. Price MR, Tillett ED, Acland RD, Nettleton GS. Determining the relationship of the axillary nerve to the shoulder joint cap- sule from an arthroscopic perspective. J Bone Joint Surg Am. 2004;86(10):2135-42.

20. Eakin CL, Dvirnak P, Miller CM, Hawkins RJ. The relationship of the axillary nerve to arthroscopically placed capsulolabral sutures. An anatomic study. Am J Sports Med. 1998;26(4):5059.

21. Rowe CR, Patel D, Southmayd WW. The Bankart procedure: a long-term end-result study. J Bone Joint Surg Am. 1978; 60(1):1-16.

22. Constant CR, Murley AH. A clinical method of functional assessment of the shoulder. Clin Orthop Relat Res. 1987;(214):160-4.

23. Kagaya K, Yoneda M, Hayashida K, et al. Modified Caspari technique for traumatic anterior shoulder instability: comparison of absorbable sutures versus absorbable plus nonabsorbable sutures. Arthroscopy. 1999;15(4):400-7.

24. Torchia ME, Caspari RB, Asselmeier MA, Beach WR, Gayari M. Arthroscopic transglenoid multiple suture repair: 2 to 8 year results in 150 shoulders. Arthroscopy. 1997;13(5):609-19.

25. Matsuki K, Sugaya H. Complications after arthroscopic labral repair for shoulder instability. Curr Rev Musculoskelet Med. 2015;8(1):53-8.

26. Owens BD, Harrast JJ, Hurwitz SR, Thompson TL, Wolf JM. Surgical trends in Bankart repair: an analysis of data from the American Board of Orthopaedic Surgery certification examination. Am J Sports Med. 2011;39(9):1865-9.

27. Miller MD. Posterior shoulder insatbility. DeLee \& Drez's orthopaedic sports medicine: principles and practices. 4th ed. Philadelphia: Elsvier, Saunders; 2014. 530.

28. Millett PJ, Braun S. The "bony Bankart bridge" procedure: a new arthroscopic technique for reduction and internal fixation of a bony Bankart lesion. Arthroscopy. 2009;25(1):102-5. 